\title{
Gobierno local, ciudad y política urbana
}

\author{
Quim Brugué* \\ Ricard Gomà*
}

El artículo ofrece, desde la perspectiva disciplinaria de la ciencia politica, una propuesta de renovación conceptual en el campo de la política urbana. En primer lugar, los autores revisan las principales aportaciones teóricas de las ciencias sociales a la cuestión urbana. Sostienen que tras los desarrollos ecológicos y culturalistas, el análisis urbano entra en unaifase de involución teórica y sobrefragmentación empirica sólo superada hacia finales de la década de los setenta por las propuestas de la Escuela Urbana de París y sus seguidores británicos durante los ochenta. En segundo lugar, a partir. de la critica a la nueva teorización proponen una reconstrucción de la politica urbana sobre dos bases: a) la definición del sistema urbano como el abanico de relaciones sociales que giran en tomo a la generación, distribución de usos y asignación social del suelo y del parque inmobiliario, $y$ b) su conexión con la esfera estatal por medio de la dimensión urbana de las políticas públicas del Estado de bienestar. Finalmente, exploran el nuevo potencial de las políticas urbanas en un contexto de reforzamiento de los gobiernos locales y de transformación de las ciudades hacia un esquema postindustrial difuso.

\section{La cuestión urbana en las ciencias sociales: principales aportaciones teóricas}

La reflexión sobre la cuestión urbana desde las ciencias sociales ha estado históricamente marcada por la dificultad de delimitar un objeto de estudio sustantivo y territorialmente definido, es decir, un proceso social concreto y una categoría espacial específica: la ciudad. La articulación de estas dos dimensiones ha provocado tensiones analíticas enormes. Los intentos de teorizar sobre la cuestión urbana privilegiando uno de los dos componentes han implicado, de hecho, la disolución del otro y, por lo tanto, el fracaso en la construcción de un objeto urbano de reflexión. Esta dificultad además ha atravesado dos contextos históricos para los estudios urbanos: la fase que arranca con los procesos urbanizadores del siglo XIX, con predominio de las fuerzas del mercado en la configuración de la ciudad, y la fase que arranca con la aparición del Estado de bienestar keynesiano (EBK), con centralidad de las variables de política pública. En el primero de los contextos históricos reseñados se desarrollan los dos paradigmas urbanos clásicos: el ecológico y el culturalista.

\footnotetext{
* Profesor titular de Ciencia Política de la Universidad Autónoma de Barcelona.
} 
Los autores de la escuela ecológica clásica de Chicago entienden la ciudad como un patrón determinado de asentamiento y usos del suelo, cuyas variables causales hay que situarlas en el plano de lo ecológico: en el accionar humano provocado por recursos naturales escasos y por la superación de posibles crisis ambientales. El espacio-ciudad sería, en definitiva, un modelo específico de solución a los problemas de la relación comunidad-entorno (Lezama, 1993). En un orden secundario, la escuela de Chicago incorpora una lectura social de la ciudad: se considera a la urbanización capitalista como desencadenante de un ritmo vertiginoso de cambio social. La racionalidad especulativa pasa a ser la lógica de interacción urbana predominante, lo cual rompe los mecanismos de cohesión social comunitaria y empuja a las personas a la inestabilidad.

Los autores de la escuela culturalista entienden la ciudad como una plasmación cultural específica, expresada en un modelo de relaciones sociales definidos por el encuentro de tres rasgos: $a$ ) el carácter superficial, puntual y segmentado de las interacciones humanas; $b$ ) una ubicación personal compleja en el medio social que provoca inseguridad e inestabilidad, y $c$ ) una interacción social basada en roles específi$\cos$, debilitadora de los contactos primarios y reforzadora del instrumentalismo como actitud predominante. En el paradigma culturalista, el carácter esencialmente urbano de tal modelo social está dado por el hecho de atribuir toda la eficacia causal a tres factores que definen a la ciudad en el plano espacial: su gran tamaño, su elevada densidad y la heterogeneidad que resulta de ambas (Bettin, 1982).

En definitiva, las teorías ecológica y culturalista representan el primer intento de reflexión global sobre lo urbano, bajo condiciones de urbanización capitalista prekeynesiana. La primera define la ciudad en el plano espacial, como sistema ecológico; la segunda lo hace en el plano social, como configuración cultural. Ambas son vulnerables por motivos diferentes. La escuela de Chicago fuerza demasiado su símil biológico: ni la esencia de la ciudad se agota en los procesos de adaptación al medio, ni éstos son específicos de los asentamientos urbanos. La escuela culturalista no logra establecer conexiones causales convincentes entre la ciudad como modo de vida y sus hipotéticos determinantes espaciales. ${ }^{1}$ 


\section{La ciudad y el Estado de bienestar; emergencia de la política urbana y repliegue teórico}

Bajo condiciones de expansión del EBK, el diseño multinivel de políticas públicas de incidencia sobre la ciudad, los nuevos roles municipales de bienestar y la construcción del arsenal conceptual del policy analysis, brindan la base material y analítica necesaria para un salto cualitativo en los estudios urbanos. El rendimiento de ese posible salto adelante es desigual y presenta tres características: la irrupción y consolidación de la ciencia política en la reflexión urbana; la ausencia de propuestas renovadas de construcción teórica; y la multiplicación del volumen de investigación empírica (Judge, Stoker, Wolman, 1995). El repliegue teórico, articulado al avance en la producción politológica de estudios de caso, genera un uso enormemente fragmentado de la categoría política urbana. Williams (1971) resume con precisión el giro ecléctico y pragmatista:

Urban is a catch-all adjective loosely used to classify a bloc of heterogenous studies that have little or no theoretical affinity (...) Orientations among political scientists towards urban politics involve a variety of structures, processes and policy areas commonly found in cities. While there is nothing intrinsically wrong with these approaches... there is nothing that is explicitly urban about them (Williams, 1971: 5-6).

Profundizando en el argumento de Williams, puede observarse cómo, durante la fase de expansión del EBK, el concepto de política urbana se utiliza en dos sentidos: como equivalente a programa de actuación del gobierno local de las ciudades (priorización del criterio institucional) y como equivalente a intervención pública sobre aspectos relacionados con la naturaleza de las ciudades, al margen del nivel institucional de diseño de la política (priorización del criterio sustantivo). Ambos usos confluyen en una muy débil e incluso inexistente fundamentación teórica de la cuestión urbana. La única fuente de recursos teóricos es externa al ámbito de la reflexión urbana y viene dada por la proyección de la emergente teoría de políticas públicas al área de las políticas urbanas (como quiera que éstas fueran definidas). Cabe aquí destacar el largo y fértil debate sobre las es-

sentan quizás los mejores estudios sobre los paradigmas clásicos traducidos al castellano. Como fuentes originales, también traducidas, véase Park (1988) (escuela ecológica) y Wirth (1988) (escuela culturalista). 
tructuras de poder comunitario que enfrenta a las corrientes pluralista y elitista, y las aportaciones de la escuela neoweberiana, ${ }^{2}$ que focaliza sus estudios en el rol estratégico de las burocracias locales como verdadera llave en la asignación de recursos públicos entre colectivos y necesidades urbanas en competencia.

La reteorización política de la cuestión urbana: ciudad y reproducción social de la fuerza de trabajo

A partir de los años setenta se produce un giro fundamental en la reflexión sobre la cuestión urbana desde las ciencias sociales. Se recupera el hilo de la construcción teórica, interrumpido por el predominio empiricista de las décadas anteriores. Tal renovación teórica es llevada a cabo por la escuela neomarxista de París, con aportaciones claves de autores como Castells (1974), el cual replantea la cuestión urbana estrechamente ligada a la reproducción social de la fuerza de trabajo, y Lojkine (1979), (re)teorizador del proceso urbano como generador de condiciones socioespaciales de acumulación. Durante los años ochenta se produce una fecunda relaboración de los postulados franceses por parte de la izquierda urbana inglesa, destacando la teoría de los consumos colectivos de Dunleavy (1980) y la tesis del Estado dual de Saunders (1986). Tal conjunto de aportaciones no sólo resitúan el análisis urbano en una perspectiva teórica global, sino que lo hacen destacando la centralidad de las variables políticas e insertando los nuevos paradigmas en la tradición acumulada de investigación empírica. ${ }^{3}$

Todo ello abre perspectivas a la construcción, por vez primera, de una reflexión urbana basada en la articulación teórico-empírica en el marco disciplinario de la ciencia política. Sintetizar el conjunto de las aportaciones citadas desbordaría los objetivos de este capítulo. Se intentará, sin embargo, una breve síntesis de la teoría de los consumos colectivos, quizás el filón argumental que ha generado un debate aca-

${ }^{2}$ El libro de Judge, Stoker y Wolman (1995) presenta una magnífica revisión crítica del rendimiento teórico de las escuelas pluralista, elitista y neoweberiana aplicadas a los estudios urbanos. Como fuentes originales, véase Dahl (1961), Hunter (1953), Polsby (1980) y Pahl (1975).

Como precedente de la escuela urbana neomarxista de París debe citarse a Lefebvre (1978). A él se vinculan, de una u otra manera, todas las aportaciones posteriores de Castells y Lojkine. 
démico más amplio. La crítica a los aspectos más vulnerables de dicha teoría abrirá paso a nuestra propuesta conceptual de política urbana.

La teoría urbana de los consumos colectivos

La teoría de los consumos colectivos (TCC) abandona toda perspectiva de definición de la cuestión urbana a partir de variables de carácter espacial. La tesis de fondo es bien clara: las estructuras espaciales están desprovistas de valores causales en relación con determinados procesos sociales que cristalizan en el espacio urbano. Tales procesos no son más que la expresión de otro tipo de aspectos de fondo, inscritos en el núcleo del capitalismo avanzado. A partir de aquí el objeto urbano se reformula de manera radical. La significación teórica de la ciudad pasa por su consideración como connotación territorial específica de un proceso social: las relaciones de consumo conectadas a la reproducción de la fuerza de trabajo. Es decir, el sistema urbano como unidad espacial de reproducción social de la población.

Los autores de la TCC constatan que, en el contexto del Estado de bienestar keynesiano, se extiende de forma masiva la implicación de las administraciones en la provisión de valores de uso, en forma de servicios de acceso universal. Sobre esta base, el ámbito de la política urbana queda enmarcado, precisamente, por la toma de decisiones públicas sobre los procesos de consumo colectivo. Como muestra el cuadro 1 , Dunleavy (1980) construye un esquema donde se establecen los criterios que permiten situar a un proceso sociopolítico en el terreno de los consumos colectivos. En primer lugar propone una distinción nítida entre las esferas de la producción (donde se obtienen salarios a cambio de trabajo o se asignan rentas por decisión pública) y la esfera de la reproducción (donde se desarrollan relaciones de consumo de servicios y mercancías). En segundo lugar fija de forma progresiva el ámbito de la política urbana, vinculando ésta a los procesos de consumo de servicios públicamente regulados y gestionados, ofrecidos con criterios de acceso no mercantiles o financiados por vía impositiva. Esta concreción conceptual permite a Dunleavy detallar los ámbitos de actuación que comprende la política urbana: vivienda, transporte, infraestructuras viales, sanidad, educación y servicios sociales. 
CUADRO 1

Ámbito sustantivo de la política urbana según la TCC

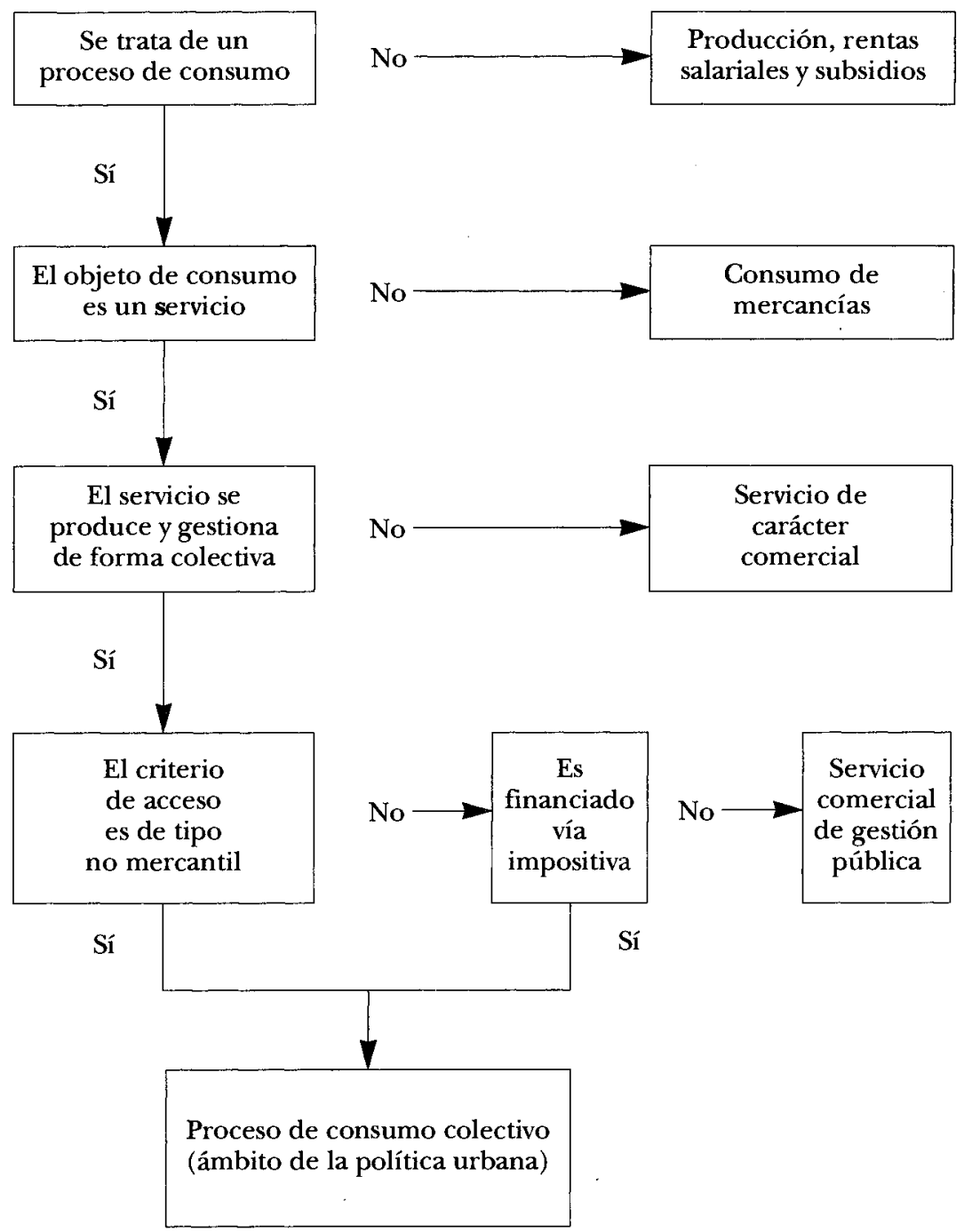

Fuente: Dunleavy (1980: 53). 
Hacia una reconstrucción teórica de la política urbana

La tesis de los consumos colectivos tiene dos puntos de gran relevancia: su voluntad de articulación teórico-empírica y de consolidar las reflexiones sobre la cuestión urbana en la ciencia política reciente. Mantiene, sin embargo, mal resuelto el problema histórico de conceptualizar la política urbana a partir de la confluencia de lo espacial y lo social. La TCC desplaza el ámbito de análisis urbano hacia los procesos sociopolíticos, a la vez que abandona cualquier intento de reflexión territorial. Llegados a este punto intentaremos una nueva reconstrucción teórica de la política urbana: en primer lugar acotaremos conceptualmente el sistema urbano, posteriormente estableceremos su vinculación con el Estado por medio de las políticas públicas.

\section{La articulación territorial de los modelos socioeconómicos}

Cada gran ciclo de desarrollo de las sociedades modernas -con sus respectivos esquemas de crecimiento, distribución y consumo- presenta unas determinadas pautas de proyección territorial; pautas que, siguiendo a varios autores, englobaremos en el concepto de división económica y social del espacio (Derycke, 1982). Veamos en detalle los dos componentes de dicho concepto.

La división económica del espacio: la articulación territorial de los sistemas productivos

La conformación de espacios productivos tiene que ver con la progresiva desvinculación de la actividad económica respecto de condicionantes geonaturales, y la necesidad de reconexión funcional de ésta-como factor de competitividad-a medios técnicos integrados, en el marco de los cuales se originan economías de aglomeración, colectivos laborales cualificados y redes de relaciones interempresariales. Dada la vinculación de los espacios productivos a sus contextos histórico-económicos globales y dado el carácter cíclico del desarrollo capitalista, podemos sostener la idea de que cada cambio de modelo de acumulación genera una dinámica simultánea de restructuración territorial.

Bajo condiciones fordistas, la división económica del espacio se asienta sobre tres aspectos fundamentales: el carácter manufacturero de las bases económicas urbanas, el predominio de unidades integra- 
das que fabrican en gran escala bienes estandarizados, y la naturaleza masivo-periférica del proceso industrial, configurador de coronas metropolitanas primarias. La quiebra del fordismo se traslada al territorio e implica el colapso del esquema vigente de funcionamiento económico de la ciudad. De forma simultánea a una profunda recesión en la construcción, se desmantelan los tejidos industriales clásicos, con la consiguiente destrucción masiva de ocupación urbana.

E1 reciente ciclo expansivo genera nuevas bases de organización de los espacios productivos. ${ }^{4}$ En primer lugar, se consolida la expansión del terciario de alto valor añadido, acompañada de una reactivación muy selectiva en la industria y la construcción. En segundo lugar, las relaciones espacio-actividad económica quedan sujetas a un doble proceso de cambio: recentralización del terciario avanzado y de las funciones direccionales del aparato productivo, y desconcentración industrialocupacional hacia la segunda corona, marco de la especialización flexible. Finalmente, la transformación fundamental quizás pase por la neocentralidad del territorio como motor del desarrollo endógeno y por la extensión cualitativa del rol económico de la ciudad, como nudo direccional básico del nuevo modelo global (Castells, 1989).

La división social del espacio: vivienda, consumos colectivos y estratificación urbana

La transferencia de la estructura social al territorio se realiza fundamentalmente por medio de la producción mercantilizada de suelo y vivienda. Ambos mercados tienen un funcionamiento muy específico en relación con otros mercados privados de bienestar: operan interconectados (con subordinación del primero al segundo), presentan una concurrencia muy imperfecta y una elevada carga especulativa. En ellos entran en juego diferentes actores con intereses en conflicto y distribuciones enormemente asimétricas de recursos. Los valores que se forman -las rentas del suelo y su articulación con los precios inmobiliarios- operan como instrumentos de distribución entre usos y de asignación entre grupos sociales. ${ }^{5}$ Las consecuencias son bási-

${ }^{4}$ Sobre procesos concretos de restructuración económico-urbana pueden consultarse los trabajos sobre Nueva York, Londres y París de Savitch (1991) y Fainstein et $a l$. (1992). Para el caso de Barcelona, véase Trullén (1991), Ferrer y Nel.lo (1991).

${ }^{5}$ Para una revisión en profundidad del concepto de renta urbana en todas sus variantes, asî como de sus impactos sobre la segregación social espacial, véase Harvey (1977) y Topalov (1984). 
camente dos: por una parte, la segregación urbana definida en sus múltiples vectores: centro-periferia, escisión de usos y segregación social en el espacio; por otra parte, la crisis estructural de la vivienda, con exclusión del mercado inmobiliario de amplios sectores de demanda insolvente. Como en el caso de los espacios productivos, los espacios habitacionales se vinculan también a la dinámica de sus contextos globales. Veamos las pautas de cambio en la división social del espacio en relación con las grandes transformaciones recientes.

La ciudad industrial conecta el crecimiento demográfico acelerado -vinculado a grandes migraciones- con un desarrollo habitacional masivo, periférico y segregado. La vivienda se estandariza, como todos los procesos de consumo bajo condiciones fordistas, y se rompe el modelo de complejidad urbana de usos, el cual queda relegado, y bajo fuertes presiones, a los centros históricos (Campos Venutti, 1981). Desde los años setenta, la crisis del modelo económico supone también la parálisis del urbanismo desarrollista y deja al descubierto enormes desequilibrios e insuficiencias: infravivienda, subequipamiento y sobredensidad. De forma reciente se abren paso nuevos modos de organización del espacio habitacional, coherentes con los aspectos centrales del postindustrialismo. Por un lado, la reversión demográfica de las primeras coronas metropolitanas se contrapone a la expansión de nuevas áreas externas, menos densificadas y con nuevas pautas clientelizadas de consumo de vivienda: sustitución del bloque por la casa unifamiliar o nuclear. Por otro lado, la dualización de los mercados privados de bienestar se manifiesta también en el territorio, con un gran crecimiento del volumen de exclusión poblacional respecto de la oferta mercantil de viviendas. Por último, se intensifica la transformación de los tejidos urbanos centrales. Remergen los conflictos de usos y se acelera la sustitución: los colectivos de rentas altas tienden a apropiarse de los valores de centralidad, restableciéndose así la lógica de la estratificación social urbana (Alabart et al., 1994).

Dos dinámicas de actuación sobre el espacio: urbanización y remodelación

Hemos sostenido que en cada fase histórica el régimen de acumulación y la estructura de clases presentan una determinada articulación territorial definida por las pautas de localización de las actividades económicas (directivas y productivas) y por las fracturas sociales del espacio derivadas de los precios del suelo y la vivienda. En cada uno 
CUADRO 2

La articulación territorial de los modelos socioeconómicos

\begin{tabular}{|c|c|c|c|c|}
\hline \multicolumn{2}{|c|}{$\begin{array}{l}\text { Modelo económico } \\
\text { Estructura social }\end{array}$} & \multicolumn{2}{|c|}{$\begin{array}{l}\text { División económica } \\
\text { y social del espacio }\end{array}$} & \\
\hline \multirow[t]{2}{*}{$\begin{array}{r}\text { Divi } \\
\text { del } e \\
\\
\text { El modelo } \\
\text { genera pau } \\
\text { de los espa }\end{array}$} & $\begin{array}{l}n \text { económica } \\
\text { acio urbano } \\
\text { acumulación } \\
\text { s de organizac } \\
\text { os productivos }\end{array}$ & \multicolumn{3}{|c|}{$\begin{array}{l}\text { División social } \\
\text { del espacio urbano } \\
\text { odelo social se traslada } \\
\text { rritorio en términos de } \\
\text { atificación residencial }\end{array}$} \\
\hline & $\begin{array}{l}\text { Capitalismo } \\
\text { industrial }\end{array}$ & $\begin{array}{c}\text { Capitalismo } \\
\text { postindustrial }\end{array}$ & $\begin{array}{c}\text { Estructura } \\
\text { social } \\
\text { de clases }\end{array}$ & $\begin{array}{c}\text { Estructura } \\
\text { social } \\
\text { fragmentada }\end{array}$ \\
\hline $\begin{array}{l}\text { Producción } \\
\text { de nuevo } \\
\text { espacio } \\
\text { urbano }\end{array}$ & $\begin{array}{l}\text { Metropoliza- } \\
\text { ción industrial } \\
\text { intensiva } \\
\text { en empleo }\end{array}$ & $\begin{array}{l}\text { Crisis y } \\
\text { reactivación } \\
\text { industrial } \\
\text { selectiva. } \\
\text { Terciarización } \\
\text { del empleo } \\
\text { no cualificado }\end{array}$ & $\begin{array}{l}\text { Periferización } \\
\text { habitacional } \\
\text { masiva y } \\
\text { homogénea }\end{array}$ & $\begin{array}{l}\text { Proceso } \\
\text { urbanizador } \\
\text { de nuevo } \\
\text { tipo: difuso y } \\
\text { diversificado }\end{array}$ \\
\hline $\begin{array}{l}\text { Remodelación } \\
\text { del espacio } \\
\text { consolidado }\end{array}$ & $\begin{array}{l}\text { Desmantela- } \\
\text { miento de } \\
\text { los tejidos } \\
\text { industriales } \\
\text { tradicionales }\end{array}$ & $\begin{array}{l}\text { Terciarización } \\
\text { avanzada } \\
\text { de zonas } \\
\text { con altos } \\
\text { valores de } \\
\text { centralidad }\end{array}$ & $\begin{array}{l}\text { Espiral de } \\
\text { degradación } \\
\text { de vivienda, } \\
\text { equipamientos } \\
\text { y espacios } \\
\text { colectivos }\end{array}$ & $\begin{array}{l}\text { Regeneración } \\
\text { habitacional } \\
\text { selectiva con } \\
\text { presiones de } \\
\text { sustitución } \\
\text { social y } \\
\text { funcional }\end{array}$ \\
\hline
\end{tabular}


de esos modelos territoriales es posible distinguir dos tipos de actuaciones de los agentes: la producción extensiva de nuevo espacio urbano (incorporación de suelo al proceso urbanizador), y la restructuración de los tejidos ya consolidados, generadora de un abanico de impactos sociales, morfológicos y funcionales. Ambos tipos de intervención pueden afectar a los componentes económicos y habitacionales del territorio. Repasemos, a modo de ejemplo, las grandes pautas evolutivas de estos últimos.

El modelo de urbanización del capitalismo liberal se caracterizó por la producción y consolidación de los ensanches, como espacios de apropiación social burguesa, y por la restructuración de ciertas zonas estratégicas del tejido histórico preindustrial (López, 1993). El modelo del capitalismo keynesiano articuló la producción de periferias extensivas con el desarrollo simultáneo de una espiral de degradación en los centros históricos. Su crisis supuso la ruptura de la lógica desarrollista y el diseño inicial de procesos de regeneración sobre la ciudad consolidada. Finalmente, el modelo urbano postindustrial implica, por un lado, el restablecimiento de una lógica de crecimiento, esta vez de tipo difuso y diversificado, así como la reaparición de fuertes presiones sobre los espacios centrales, tendentes a la terciarización y la fijación de usos residenciales de elevada rentabilidad. Estos últimos, palanca del recambio poblacional en beneficio de segmentos de clase media y alta.

La política urbana como regulación pública de la división económica y social del espacio

En síntesis, hemos acotado la dimensión espacial del problema urbano en la confluencia del ordenamiento territorial del régimen de acumulación con la forma de distribuir el espacio habitacional entre grupos y clases sociales. Yhemos sostenido que ambos procesos operan, bajo condiciones históricas concretas, sobre la producción de nuevo espacio urbano y sobre el remodelamiento del existente. A partir de aquí podemos lanzar una tentativa de definición del sistema urbano como el abanico de relaciones sociales que giran en torno a la generación, distribución de usos y asignación social del suelo y del parque inmobiliario. El siguiente paso en la línea argumental consiste en interconectar el sistema urbano a la esfera del Estado y, en concreto, a la elaboración de políticas públicas que inciden sobre los procesos urbanos, tal como han sido definidos. Dicha interconexión nos permitirá delimitar sustantivamente el concepto de po- 
lítica urbana. Para esta labor contamos con dos puntos de arranque: las contradicciones urbanas y los valores políticos del Estado de bienestar.

Las contradicciones urbanas del capitalismo avanzado

En el marco del capitalismo avanzado, la actuación de las fuerzas del mercado sobre el sistema urbano genera contradicciones en su doble vertiente económico-ocupacional y sociorresidencial.

La división económica del espacio afronta dos tipos de problemas internos. En primer lugar, los derivados del impacto de las crisis generales sobre las bases productivas y los mercados laborales urbanos, los cuales se resuelven cíclicamente en difíciles y costosos cambios estructurales en los esquemas de funcionamiento económico de las ciudades. En segundo lugar, los problemas vinculados a los límites inherentes al mercado como mecanismo de regulación social y que, en este caso, se expresan en un mínimo de tres direcciones: los conflictos interfuncionales (por ejemplo, el terciario avanzado frente a la residencia central); la eficiencia general del sistema frente a las estrategias individuales de localización, y las preferencias del capital productivo frente a los intereses de la propiedad urbana (por ejemplo, en materia de mercado de alquileres). Todas estas contradicciones ofrecen espacios potenciales de actuación pública.

La división social del espacio afronta, por su parte, dos tipos adicionales de contradicciones internas. En primer lugar, el impacto de la segregación de usos sobre la calidad residencial, el cual genera, por un lado, défícit localizado y selectivo de infraestructura y equipamiento urbano y, por el otro, necesidades de desplazamiento y demandas sociales de transporte no siempre rentables para ser cubiertas desde la lógica del mercado. En segundo lugar, los problemas vinculados a los límites del mercado como mecanismo de producción y asignación de viviendas, los cuales se expresan en tres direcciones básicas: la crisis estructural de oferta, dadas la rigideces del sector y sus inelasticidades en la dinámica evolutiva de la demanda; el carácter socialmente excluyente de los precios de mercado, y la explotación diferencial, en ese mercado, de los grupos de rentas más bajas por medio de la reducción de excedentes de consumidor. ${ }^{6}$ Todas estas

\footnotetext{
${ }^{6}$ El excedente del consumidor es la diferencia entre lo que una persona paga realmente por un bien y lo que querría pagar con tal de no pasar sin él. Aplicado a la vivienda sería la diferencia entre intención máxima de gasto y precios de mercado de
} 
contradicciones generan nuevos espacios posibles de actuación pública. Su concreción o no en forma de políticas urbanas dependerá de los valores ideológicos predominantes en la esfera gubernamental y en la red de actores sociales con capacidad de influencia.

\section{La dimensión urbana del Estado de bienestar}

En las democracias capitalistas avanzadas, la organización política internaliza parcialmente el conflicto social. La regulación y gestión de buena parte de las contradicciones se desplaza a la esfera del Estado. Las políticas públicas aparecen, en consecuencia, como espacios centrales (a veces únicos) de mediación y resolución de conflictos entre colectivos con preferencias no coincidentes. En el marco del EBK y de sus procesos de restructuración no desmanteladores se erigen dos macroespacios de intervención pública. El primero conformado por el conjunto de instrumentos de gestión política del crecimiento, es decir, por las políticas públicas que dotan de un cierto nivel regulativo al sistema económico, en sus dimensiones monetaria, productiva y laboral. El segundo conformado por el abanico de políticas sociales (servicios y transferencias monetarias) que incide sobre el conflicto distributivo, es decir, sobre la asignación de los recursos entre grupos y clases sociales.

En este contexto, los problemas urbanos anteriormente señalados son insertados también en un amplio proceso de mediación política por parte del Estado. La proyección al sistema urbano de la capacidad de intervención del Estado de bienestar implica la politización -a mayor o menor escala- de las contradicciones inherentes a la división económica y social del espacio (DESE). La agenda de actuación del Estado amplía por tanto su extensión material, y las políticas públicas se convierten, también, en espacios de regulación y gestión del conflicto urbano. Siguiendo el razonamiento anterior podemos sostener que las políticas urbanas implican la expansión del Estado de bienestar (EB) hacia un tercer macroámbito de intervención definido en clave espacial, pero vinculado a los procesos de crecimiento y distribución de la riqueza. Utilizando el modelo de Offe (1990), las políticas urbanas completarían la vinculación del Estado con sus sistemas de flanco (económico y social); así, la proyección de éstos en el espacio traería consigo el dise- 
CUADRO 3

La política urbana: como dimensión espacial del EB y como forma de regulación política de la DESE

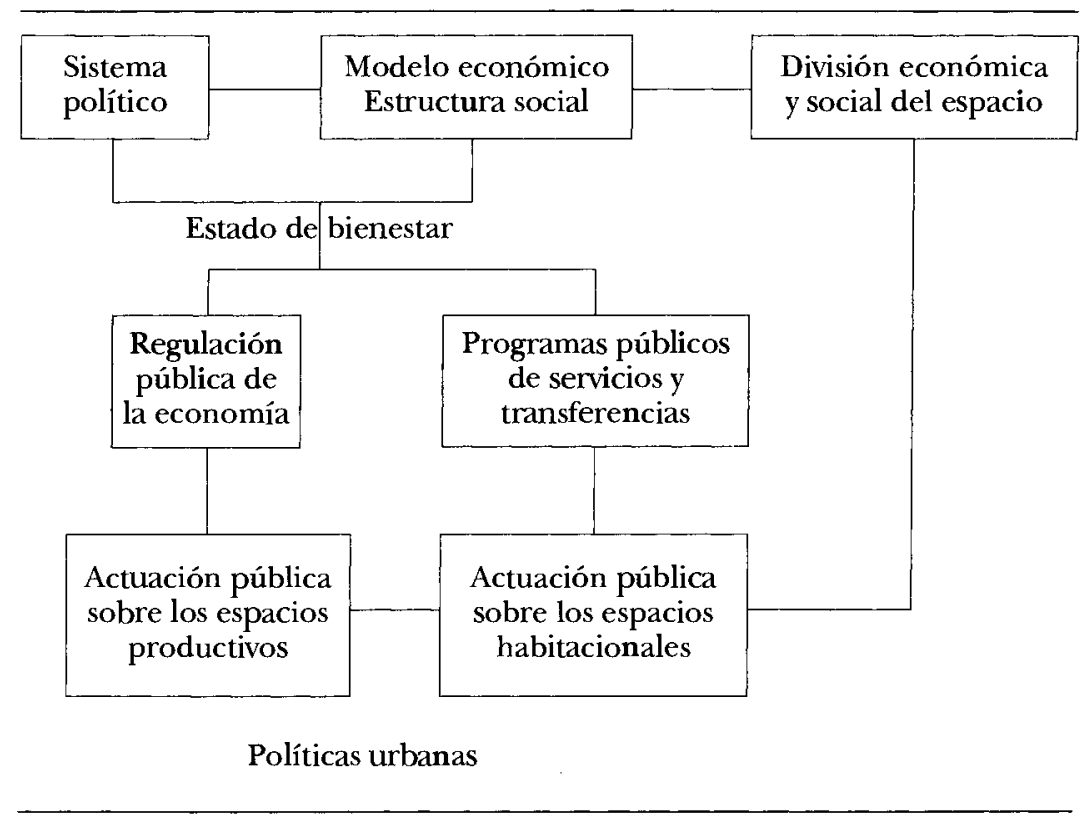

ño de políticas urbanas, en tanto que son espacialización de las políticas económicas y sociales.

En síntesis, y revisando a Dunleavy, el ámbito sustantivo de la política urbana -como dimensión espacial del EB o como forma de regulación pública de la DESE- incluiría los siguientes sectores de actuación pública: la localización y restructuración económica del territorio, la regulación de usos por medio de la planeación, la vivienda en todos sus aspectos, el transporte colectivo y los equipamientos e infraestructura conectados a la oferta de servicios públicos.

\section{Gobiernos locales y nuevas políticas urbanas}

Los gobiernos locales presentan una práctica política enormemente contingente, determinada tanto por factores internos (valores políticoideológicos de las mayorías municipales y estructuras socioeconómicas del municipio) como por factores externos (modelo de bienes- 
tar predominante y esquema de distribución territorial del poder del Estado). Sin embargo, es posible extraer algunas regularidades y tendencias generales en torno a los gobiernos locales del EBK, en tanto que son precedente inmediato de las nuevas aportaciones locales a las políticas urbanas de los noventa.

\section{El municipio de bienestar en transición: nueva agenda y nuevos roles}

Adoptemos, como punto de arranque analítico la tesis del Estado dual (TED) propuesta por Saunders a finales de los setenta. Según la TED, ba:jo condiciones de expansión keynesiana y consenso socialdemócrata, se desarrolló en Europa un patrón nítido de división política de roles entre los estados centrales y los poderes locales. Los primeros regulaban la esfera de la producción, donde las fuerzas del mercado mantenían su supremacía, gestionaban intereses de clase y lo hacían por medio de mecanismos corporativistas. Los poderes locales, por el contrario, tendieron a orientar su actuación hacia las políticas sociales, con predominio de los valores de servicio público y en un marco mucho más pluralista de actores, intereses e interacciones. Si se observa más en detalle la estructura de políticas públicas que presentan las agendas locales, aparecen dos rasgos en primer plano. Por un lado, los municipios de bienestar se especializan en la oferta de servicios públicos universales y en la regulación del proceso de expansión urbana, sin ninguna aportación relevante en el ámbito de la promoción económica. Por el otro, los roles que esos gobiernos locales juegan en esos ámbitos son operativos, ejecutores -a gran escala- de estrategias que ellos no diseñan.

Durante los últimos diez o quince años se producen cambios socioeconómicos y urbanos de gran alcance que impactan y alteran de raíz el escenario de políticas y roles locales prexistente. En concreto, el triple cambio hacia estructuras sociales mucho más fragmentadas, hacia un modelo económico más valorizador del territorio y hacia un sistema urbano difuso con nuevos ejes de segregación, se proyecta en una agenda local más compleja y en nuevos roles poláticos municipales, más cualitativos y estratégicos. Todo ello en un marco de nuevas confluencias intergubernamentales, donde las pautas de división central-local del trabajo entran en crisis terminal.

Las políticas locales de bienestar social intentan dar respuesta a los nuevos ejes de desigualdad y a la nueva cultura posmaterialista del bienestar, articulando programas focalizados de acción positiva con estrategias de dinamización comunitaria. Las políticas locales de de- 
sarrollo económico -por medio de un abanico de iniciativas de desarrollo- pretenden hacer del territorio el eje básico de un proyecto económico, donde actores públicos, privados y asociativos confluyan en un horizonte estratégico. Veamos ahora con más detalle cómo la fase posdesarrollista de cambio urbano no sólo genera nuevos modelos espaciales, sino también una redefinición en profundidad de las políticas urbanas y de la posición de los poderes locales.

\section{La ciudad postindustrial difusa: hacia una nueva división económica y social del espacio urbano}

La salida de la crisis del modelo industrial-desarrollista supone quizás la restructuración más profunda de la gran ciudad desde el impulso urbanizador del siglo XIX. Las ciudades postindustriales ya no presentan límites geográficos nítidos, ni un esquema espacial polarizador de un único centro y sus respectivas periferias suburbanas. Son territorios de geometría variable, donde se desarrollan, con densidades e intensidades desiguales, redes de relaciones económicas, ocupacionales, culturales, de ocio, asociativas, o de asistencia informal. Presentan una espacialización de tipo urbano-regional, difusa, de carácter polinuclear, donde a la estructura radial expresiva de las viejas polarizaciones se empieza a superponer una estructura mucho más reticular como reflejo del entramado de relaciones multilaterales entre los diversos centros. Estos sistemas urbanos tienden a presentar un gran dinamismo. Cuando las ciudades se convierten en nudos direccionales de la economía global y la volatilidad del capital aumenta, las ventajas competitivas de tipo local se incrementan proporcionalmente (Nel.lo, 1990). Por ello, las ciudades se ven empujadas a operar como centros de atracción-retención económica por medio de procesos de adaptación infraestructural permanente. En definitiva, crecen las complejidades y las potencialidades del sistema urbano, muy por encima de las épocas recientes.

Pero eso no es todo. La dialéctica urbana no cesa y a los nuevos potenciales debe añadirse un conjunto de nuevas contradicciones. Las nuevas pautas de localización productiva y terciaria tienden a incrementar la fractura económica del espacio: mientras los servicios a las personas y las ocupaciones menos cualificadas se periferizan, el terciario avanzado y la toma de decisiones industriales refuerzan centralidades excluyentes. Los mer- 
cados laborales urbanos se desinstitucionalizan y se segmentan. El incremento global de la renta disponible se hace compatible con el incremento de la segregación espacial de los grupos sociales. Se generan procesos de movilidad residencial forzada por las dificultades e imposibilidades de acceso a la vivienda (Nel.lo, Valles, 1992). Y, finalmente, la renta urbana de la propiedad del suelo presiona como mecanismo de remodelación de los centros históricos, por encima de sus valores de uso.

\section{La dimensión local del gobierno y la gestión pluralista urbana}

Así pues, dos macroprocesos confluyen hacia la revalorización de las políticas urbanas y de su dimensión local. Por una parte, los gobiernos locales tienen la oportunidad de convertirse en coprotagonistas de un Estado de bienestar restructurado, mucho más cercano a valores comunitarios que el modelo keynesiano. Por la otra, las políticas urbanas tienen ante sí el reto de diseñar y poner en práctica nuevos y potentes instrumentos de regulación pública de la ciudad difusa, si no se quiere que ésta sea el resultado estricto de la lógica del mercado.

El abanico de las nuevas políticas urbanas

De esta confluencia deben nacer los perfiles de una estrategia territorial pluralista, de fuerte componente local, para articularse mediante un abanico de políticas urbanas como reflejo de ciertos valores de fondo.

a) Los valores marco de la estrategia territorial podrían formularse de la siguiente manera:

- La decisión colectiva debería predominar sobre la renta urbana como mecanismo de regulación global de la ciudad.

- El territorio debería ser un escenario de diálogo paritario en tre eficiencia económica, equidad y redistribución social.

b) Los principios y objetivos que despliegan los anteriores valores podrían concretarse de la siguiente forma:

- Equilibrio económico espacial: difusión de centralidades.

- Derecho universal a transporte, vivienda y servicios.

- Acción positiva contra la segregación socioespacial.

- Complejidad funcional: articulación de usos diversos.

c) Tales principios y objetivos podrían hacerse tangibles por medio del siguiente cuadro de políticas urbanas: 
Las politicas urbanas de la eficiencia: a) creación de nuevos espacios de terciario avanzado, directivas y de zonas integradas de actividades logísticas, como instrumentos de difusión de la centralidad en el territorio; b) planeación y producción de suelos industriales y comerciales que posibiliten la diversificación de las bases económicas de la ciudad; c) infraestructura vial no generadoras de segregación poblacional ni de agresión ambiental, y con fuerte presencia pública en el suelo de nueva accesibilidad.

Las políticas urbanas de la equidad: a) municipalización de suelo para generar nuevo espacio residencial, incidir sobre los precios de mercado y debilitar las actitudes especulativas de los agentes privados; b) universales de vivienda desplegadas en un mínimo de tres dimensiones: programas de garantía de acceso mediante promociones públicas y concertadas, programas de rehabilitación, e iniciativas de diversificación del régimen de ocupación; c) equipamientos de servicios personales (centros cívicos) y universales (escuelas y cen tros de salud), como garantía de la distribución equitativa de éstos en el territorio; d) transporte público colectivo orientadas a su potenciación frente al privado individual, a la mejora de sus tasas de cobertura poblacional e intensidad de servicio, y a la integración entre diferentes medios.

Las politicas urbanas de la redistribución: a) selectivas de vivienda instrumentadas por medio de programas de vivienda adaptada y subsidios en el mercado de alquileres, y orientadas a colectivos vulnerables a procesos de exclusión social; b) actuación integral en barrios periféricos multidegradados, dirigidas a la mejora de las condiciones generales de residencia, pero también a la transformación de espacios monoclasistas y monofuncionales en espacios socialmente equilibrados y de actividades diversas; c) regeneración de los tejidos históricos dirigidas a reforzar la heterogeneidad social y funcional de dichos espacios. Las operaciones de vivienda, de equipamiento y de espacio público de uso colectivo deberían articularse con programas de servicios personales y con iniciativas de reactivación económica.

Políticas urbanas y esfera local: una relación intensa, dinámica y compleja

Los gobiernos municipales deben:jugar roles cualitativos en todas estas políticas urbanas. Sin embargo, todas ellas desbordan también las 
capacidades políticas y de gestión de los poderes locales. De aquí la enorme importancia de desplegar estrategias relacionales y estructuras de asociación. La esfera política local debería elevar sus techos competitivos y, sobre todo, su participación relativa en el ingreso y el gasto público. Debería dotarse también de unas estructuras territoriales con capacidad de articular las dimensiones comunitarias y las prestaciones, superando tanto la hiperfragmentación como el espejismo tecnocrático del macromunicipio sin bases de identidad. Ahora bien, los límites que puedan derivar de esas variables no deben servir tampoco de excusa para la no acción municipal. Los gobiernos locales deberían asumir el principio de responsabilidad general sobre el bienestar de la comunidad y a partir de ahí conjugar sus propios recursos competitivos y económicos con estrategias de concertación social e institucional de objetivos, financiamiento y líneas de acción.

La elaboración de muchas políticas urbanas se desarrolla ya en un marco de gobierno y gestión pluralista. Veamos algunos ejemplos. Las políticas económicas urbanas mejoran sus resultados de forma proporcional al reforzamiento de las estructuras de asociación público-privada. Los programas de vivienda y transporte resitúan el pluralismo en el terreno de la gobernabilidad multinivel. La regeneración de barrios degradados requiere de la participación integrada de múltiples ámbitos de acción en el seno del gobierno local. En muchos países se extiende la gestión asociativa o cooperativa de las promociones públicas de vivienda, o bien se generaliza la participación directa del capital privado en los instrumentos de gestión cuasi mercantil de las políticas de rehabilitación de los centros urbanos. En todos estos casos, el gran reto de los equipos de gobierno municipal despliega habilidades de articulación del pluralismo urbano y de capacidad de liderazgo de la red de actores implicados.

Ahora bien, los roles específicos a:jugar por los poderes locales van a depender sobre todo de variables institucionales e ideológicas. La posición político-financiera del gobierno local en el Estado, cruzada por las opciones de fondo de los equipos de gobierno municipal en el eje regulación-mercado van a determinar modelos dispares de política urbana (Gurr, King, 1987).

Durante los últimos años, el rol de la esfera municipal y de la decisión colectiva en el proceso de cambio urbano ha tendido a reforzarse en los países escandinavos y centroeuropeos. En los países mediterráneos, los avances desiguales en la voluntad política de los gobiernos locales siguen chocando con estructuras territoriales y financieras muy 
poco o nada adecuadas del gobierno urbano. Finalmente, en el régimen local británico, la doble estrategia de los gobiernos conservadores -erosión institucional de los poderes locales articulada con predominio de valores neoliberales- ha provocado la desmunicipalización masiva de la regulación urbana, bien en beneficio del mercado (privatización de la vivienda social), bien en favor de agencias estatales territorializadas (regeneración de áreas degradadas) .

\section{Sintesis y cor_slusión}

El objetivo de este apartado es recoger las ideas más relevantes que han aparecido a lo largo de este capítulo y condensar el hilo argumentai que les ha intentado dar coherencia.

1) En las democracias capitalistas avanzadas, la organización política internaliza parcialmente el conflicto social. Las políticas públicas aparecen en consecuencia como espacios centrales de mediación y resolución de conflictos entre colectivos con preferencias no coincidentes. En este contexto, los problemas urbanos son insertados también en un amplio proceso de mediación política. La proyección de la capacidad de intervención del Estado de bienestar al sistema urbano implica la politización de las contradicciones inherentes a la división económica y social del espacio. La agenda de actuación del Estado amplía por tanto su extensión material, y las políticas públicas se convierten, también, en espacios de regulación y gestión del conflicto urbano. El ámbito sustantivo de la política urbana (como dimensión espacial del Estado de bienestar o como forma de regulación pública de la división económica y social del espacio) incluiría los siguientes sectores de actuación pública: la localización y restructuración económica del territorio, la regulación de usos por medio de la planeación, la vivienda en todos sus aspectos, el transporte colectivo y los equipamientos e infraestructura conectados a la oferta de servicios públicos.

2) Durante los últimos diez o quince años se producen cambios socioeconómicos y urbanos de gran alcance que impactan y alteran de raíz el escenario de políticas y roles locales prexistente. En concreto, el triple cambio hacia estructuras sociales mucho más fragmentadas, hacia un modelo económico más valorizador del territorio y hacia un sistema urbano difuso con nuevos ejes de segregación, se proyecta en una agenda local más compleja y en nuevos roles políticos municipales, más cualitativos y estratégicos. Todo ello en un mar- 
co de nuevas confluencias intergubernamentales, donde las pautas de división central-local del trabajo entran en crisis terminal.

3) La salida de la crisis del modelo industrial-desarrollista supone la restructuración más profunda de la gran ciudad desde el impulso urbanizador del siglo XIX. Las ciudades postindustriales ya no presentan límites geográficos nítidos, son territorios de geometría variable, donde se desarrollan multiplicidad de redes de relaciones con densidades e intensidades desiguales. Su potencial aumenta de forma exponencial al convertirse en nudos direccionales de una economía globalizada de capitales volátiles. Sin embargo, nuevos ejes de segregación mantienen viva la dialéctica urbana de la ciudad capitalista: en síntesis, se desarrolla una reproducción ampliada pero de nuevo tipo de la fractura económica y social del espacio.

4) Dos macroprocesos confluyen hacia la revalorización de las políticas urbanas y de su dimensión local. Por una parte, los gobiernos locales tienen la oportunidad de convertirse en coprotagonistas de un Estado de bienestar restructurado, mucho más cercano a valores comunitarios que el modelo keynesiano. Por la otra, las políticas urbanas tienen ante sí el reto de diseñar y poner en práctica nuevos y potentes instrumentos de regulación pública de la ciudad difusa.

5) Sobre la base del principio de la decisión colectiva por encima de la renta urbana, como mecanismo de regulación general de la ciudad, es posible establecer un conjunto de políticas urbanas de eficiencia, equidad y redistribución, orientadas a satisfacer los objetivos básicos de equilibrio económico espacial; derecho universal al transporte, la vivienda y los servicios; lucha contra la segregación socioterritorial, y complejidad funcional.

6) Los gobiernos municipales deben jugar roles cualitativos en todas las políticas urbanas; sin embargo, todas ellas desbordan también las capacidades políticas y de gestión de los poderes locales. De aquí la enorme importancia de desplegar estrategias relacionales y estructuras de asociación. Los gobiernos locales deberían asumir el principio de responsabilidad general sobre el bienestar de la comunidad y a partir de ahí conjugar sus propios recursos competitivos y económicos con estrategias de concertación social e institucional de objetivos, financiamiento y líneas de actuación.

7) Los roles específicos a jugar por los poderes locales van a depender sobre todo de variables institucionales e ideológicas. La posición político-financiera del gobierno local en el Estado, cruzada por las opciones de fondo de los equipos de gobierno municipal en el eje regulación- 
mercado van a determinar modelos dispares de política urbana. La experiencia europea comparada de estos últimos años así lo demuestra.

\section{Bibliografía}

Alabart, Anna et.al. (1994), Clase, poder y ciudadanía, Madrid, Siglo XXI.

Bettin, Gianfranco (1982), Los sociólogos de la ciudad, Barcelona, Gustavo Gilli. Campos Venuti, Giussepe (1981), Urbanismo y austeridad, Madrid, Siglo XXI.

Castells, Manuel (1974), La cuestión urbana, México, Siglo XXI. (1989), La ciudad informacional, Madrid, Alianza.

Dahl, Robert (1961), Who Governs?, New Haven, Yale University Press.

Derycke, Paul Henri (1982), Economía y planificación urbanas, Madrid, Instituto de Estudios de la Administración Local.

Dunleavy, Patrick (1980), Urban Political Analysis, Londres, Macmillan.

Fainstein, Susan et al. (1992), Divided Cities, Oxford, Blackwell.

Ferrer, Amador y Oriol Nel.lo (1991), "Barcelona: la transformació d' una ciutat industrial", documento de trabajo, Barcelona, Institut d' Estudis Metropolitans de Barcelona (IEMB).

Gurr, Ted y Desmond King (1987), The State and the City, Londres, Macmillan.

Harvey, David (1977), Urbanismo y desigualdad social, México, Siglo XXI.

Hunter, Floyd (1953), Community Power Structure, Chapel Hill, UNCP.

Judge, David, Stocker y Wolman (1995), Theories of Urban Politics, Londres, Sage.

Lefebvre, Henri (1978), El derecho a la ciudad, Barcelona, Península.

Lezama, José Luis (1993), Teoría social, espacio y ciudad, México, El Colegio de México.

Lojkine, Jean (1979), El marxismo, el Estado y la cuestión urbana, México, Siglo XXI.

López, Pere (1993), Un verano con mil julios y otras estaciones, Madrid, Siglo XXI.

Nel.lo, Oriol (1990), "Polítiques urbanes en una Europa en transició", Barcelona Metrópoli Mediterrannia, Barcelona, Universidad Autónoma de Barcelona (UAB) (Quaderns Centrals, 15).

— y Josep Maria Valles (1992), "De ciutat a metròpoli", UAB (mimeo.).

Offe, Claus (1990), Contradicciones en el Estado de Bienestar, Madrid, Alianza.

Pahl, Robert (1975), Whose City?, Harmondsworth, Penguin.

Park, Robert E. (1988), "Ecología humana", en Bassols et al., Antología de sociología urbana, México, UNAM.

Polsby, Norman (1980), Community Power and Political Theory, New Haven, Yale University Press.

Saunders, Peter (1986), Social Theory and the Urban Question, Londres, Hutchinson. 
Savitch, Henry (1991), Post-Industrial Cities, Princeton, PUP.

Topalov, Christian (1984), Ganancias y rentas urbanas, Madrid, Siglo XXI.

Trullén, Joan (1991), "El planejament territorial de la Regio I des d'una perspectiva econòmica”, documento de trabajo, Barcelona, IEMB.

Williams, Williams (1971), Social Policy Research and Analysis, Nueva York, Elsevier.

Wirth, Louis (1988), "El urbanismo como modo de vida", en Bassols et al., Antología de sociología urbana, México, UNAM. 


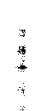

